\title{
Role of Veterinary Professionals in the Coming Time Period of Ongoing COVID-19 Pandemic
}

\section{Sanjeev Kumar*}

Department of Veterinary Microbiology, College of Veterinary Sciences and Animal Husbandry, CAU, Selesih, Aizawl, India

*Corresponding Author: Sanjeev Kumar, Department of Veterinary Microbiology, College of Veterinary Sciences and Animal Husbandry, CAU, Selesih, Aizawl, India.
Received: June 21, 2021

Published: June 29, 2021

(C) All rights are reserved by Sanjeev Kumar.
It is now more than one year since COVID-19, known as SARSCoV-2, pandemic started at the end of December 2019 in Wuhan, a central city in China. Several variants of SARS-CoV-2 were detected since then. Coronaviruses belong to the order Nidovirales in the family Coronaviridae. Coronavirinae and Torovirinae are two subfamilies of Coronaviridae family. Coronavirinae subfamily can be divided into four genera viz: Alpha-coronavirus, Betacoronavirus, Gamma-coronavirus, and Delta-coronavirus [1]. The phylogenetic analysis of the SARS-CoV-2 genome revealed that this is closely related to the Beta-coronaviruses [2]. The genome of SARS-CoV-2 is consists of positive-sense single-stranded RNA [3]. We have learned much about this virus during this period. Currently, some countries have passed or some countries are in the second wave of the COVID-19 pandemic. Globally, there have been 177,108,695 confirmed cases of COVID-19 and out of which $3,840,223$ deaths were reported to the world health organization (WHO) until 18 June 2021.

As per WHO, SARS-CoV-2 jumped the species barrier from bat to human [4]. The nose and eye is key entry portal of this virus into the body due to presence of receptor, angiotensin-converting enzyme 2, required for their entry [5]. But, these receptors are also present in the intestine and other vital organs including the heart [5]. As per WHO, symptoms are divided into three categories viz; most common symptoms, less common symptoms, and severe symptoms. The most common symptoms include fever, dry cough, and tiredness. Less common symptoms include aches and pains, sore throat, diarrhea, conjunctivitis, headache, loss of taste or smell, a rash on the skin, or discoloration of fingers or toes. Serious symptoms include difficulty breathing or shortness of breath, chest pain or pressure, loss of speech or movement.
Besides, recently many reports showed reverse zoonotic behavior (man to animals) of the COVID-19 infection in dogs, cats, minks, and large cats [6]. Covid-19 infection in dogs was reported from Hong Kong, Netherland, and the USA [7-9]. It raised serious concerns among the health community. Few cats from North Carolina, Spain, France, and Germany were found to be infected with COVID-19 [10]. The SARS-CoV-2 infection was also reported in four tigers and two lions at the Bronx Zoo in New York City [11]. In Nehru Zoological Park in Hyderabad, India, eight Asiatic lions were tested positive for Covid-19 (https://www.thehindu.com/news/cities/ Hyderabad/eight-asiatic-lions-test-positive-for-coronavirus-inhyderabad-zoo/article34480453.ece). The minks of two minks breeding farms in the Netherlands were tested positive for SARSCoV-2. All the infected animals belong to either owners or caretakers who were tested positive for COVID-19 [9].

From the above pieces of evidence of reverse zoonosis (man to animal), it can be concluded that veterinarians must keep themselves well prepared to control and prevent SARS-CoV-2 infection in the animal population in near future. There may be chances of secondary transmission from animal to human or animals may act as a reservoir of SARS-CoV-2 to humans. To date, there is no report of secondary transmission, but it is not that it may not happen in the coming time because SARS-CoV-2 was reported to be shed by infected animals. So, it is very logical to develop infrastructure and research facilities well ahead of this situation arises to diagnose and treat the infected animals, and to prevent the secondary transmission to humans. Moreover, a collaborative approach by the human medical profession and the veterinary medical profession would be better to tackle this situation. 


\section{Bibliography}

1. Elabiyi MO and Adenola OJ. "Overview of COVID-19 and way forward". Electronic Research Journal of Engineering, Computer and Applied Sciences 2 (2020): 28-36.

2. Pal M., et al. "Severe Acute Respiratory Syndrome Coronavirus-2 (SARS-CoV-2): An Update”. Cureus 12.3 (2020): e7423.

3. Astuti I and Ysrafil. "Severe Acute Respiratory Syndrome Coronavirus 2 (SARS-CoV-2): An overview of viral structure and host response". Diabetes and Metabolic Syndrome 14.4 (2020): 407-412.

4. Dhama K., et al. "SARS-CoV-2 jumping the species barrier: Zoonotic lessons from SARS, MERS and recent advances to combat this pandemic virus". Travel Medicine and Infectious Disease 37 (2020): 101830.

5. Sungnak W., et al. "SARS-CoV-2 entry factors are highly expressed in nasal epithelial cells together with innate immune genes". Nature Medicine 26 (2020): 681-687.

6. Munir K., et al. "Zoonotic and reverse zoonotic events of SARSCoV-2 and their impact on global health". Emerging Microbes and Infections 9.1 (2020): 2222-2235.

7. Sit THC., et al. "Infection of dogs with SARS-CoV-2". Nature 586 (2020): 776-778.

8. Kiros M., et al. "COVID-19 pandemic: current knowledge about the role of pets and other animals in disease transmission". Virology Journal 17 (2020): 143.

9. Subedi S., et al. "COVID-19 in Farm Animals: Host Susceptibility and Prevention Strategies". Animals 11 (2021): 640.

10. Yoo HS and Yoo D. "COVID-19 and veterinarians for one health, zoonotic- and reverse-zoonotic transmissions". Journal of Veterinary Science 21.3 (2020): e51.

11. McAloose D., et al. "From People to Panthera: Natural SARSCoV-2 Infection in Tigers and Lions at the Bronx Zoo". mBio 11.5 (2020): e02220-20.

\section{Volume 3 Issue 7 July 2021}

(C) All rights are reserved by Sanjeev Kumar. 